Colloque C2, suppl. au Journal de Physique II, Vol 1, septembre 1991

\title{
HIGH TEMPERATURE STRESS MEASUREMENTS IN CVD DIAMOND FILMS
}

\author{
C. JOHNSTON, A. CROSSLEY, A.M. JONES, P.R. CHALKER, F.L. \\ CULLEN and I.M. BUCKLEY-GOLDER
}

\author{
AEA Technology, Bullding 552, Harwell Laboratory, Didcot, \\ Oxon OXI1 ORA, Great Britain
}

\begin{abstract}
Low pressure CVD diamond films are intended for application in the optics, biomedical, electronics and engineering industries. In a number of applications, it is important to know the growth stresses in the films at room temperature and, in addition, the stresses generated in the films as a function of temperature.

The results in this paper will show the in situ measurement of stresses in CVD diamond films as a function of temperature up to $750^{\circ} \mathrm{C}$. The principal measurement techniques are Raman spectroscopic analysis and the X-ray diffraction (XRD) $\sin ^{2} \psi$ method.
\end{abstract}

\section{Introduction}

Diamond is considered to be an excellent material for many applications due to its unique range of physical and chemical properties. Not only is diamond highly transparent and exceedingly hard (it is the hardest known substance), with a high elastic modulus, it also has the highest thermal conductivity of any known material $(10-20 \mathrm{~W} / \mathrm{cmK})$ and is highly insulating with an electrical resistivity of up to $10^{16} \Omega . \mathrm{cm}$. Diamond also possesses the added advantage that it is extremely inert to chemical attack, being able to withstand very aggressive environments eg: oxygen atmospheres at $600{ }^{\circ} \mathrm{C}(1)$.

Until recently, however, diamond was only to be found in forms (stones and compacts) which limited the application to which it could be employed, viz; cutting tools/abrasives and decorative jewellery. With the development of chemical vapour deposition (CVD) processes (2) to deposit diamond thin films a whole range of further applications has been made possible in the optical, biomedical, electronics and engineering industries.

With the exceptions of cubic BN (3) and diamond itself (4), CVD diamond films deposited on heterosubstrates are polycrystalline in nature, however, their properties compare very favourably with those of the bulk material. The microstructure and morphology of diamond films is sensitive to a number of deposition parameters, such as the ratio of hydrocarbon to hydrogen used as the source gas and to the substrate temperature, to name but two. In general the films are comprised of grains with high concentrations of defects (5) such as dislocation loops and stacking faults. The films also invariably contain varying degrees of non - diamond phases such as amorphous or graphitic carbon, which may contribute to the residual stresses within the films.

Residual stresses are an important parameter which can influence coating-substrate adhesion. The residual stresses within CVD diamond thin films have been reported as both tensile and compressive in nature. Such stresses are composed of two sources, namely, intrinsic or growth stresses and thermal stresses. Structural mismatch between the film and substrate and the distribution of defects within the film are responsible for intrinsic stress while thermal stress originates from the difference in the thermal expansion coefficients of the film and the substrate.

Bower (6) has observed that in the fabrication of self-aligned rigid suspended plates of CVD diamond compressive stresses of sufficient magnitude were present to cause buckling and even rupture of plates greater than $25 \mathrm{~mm}$ in diameter. Conversely Berry et al (7) have produced diamond membranes on silicon substrates with tensile internal stresses which cause the diamond film to act as a taut layer. 
Various methods of estimating the residual stresses in diamond films have been utilised including Raman spectroscopy, X-ray diffraction analysis and mechanical methods. Boppart et al (8) have found that the Raman frequency shift $\Delta \omega / \omega$ is a linear function of strain given approximately by the relation

$$
\Delta \omega / \omega \approx-(1.0) S
$$

where $S$ is the strain and 1.0 is the approximate mode Gruneisen parameter. Yoshikawa et al (9) have used a similar approach and have concluded that CVD films deposited from ethanol/hydrogen mixtures show increasing compressive stresses with increasing concentrations of non-diamond carbon phases. The value of compressive stresses for these films was of the order of $1 \mathrm{GPa}$ with a corresponding strain of $\varepsilon=0.1-0.2 \%$ using the relationship $\varepsilon=\left(\sigma_{11}+\sigma_{12}\right) \chi$ where $\chi$ is the stress and $\sigma_{x y}$ denotes the appropriate elastic compliance constants.

Chalker et al. (10) determined the internal stresses within diamond films deposited onto silicon substrates by thermal filament CVD using the $\sin ^{2} \psi$ method of X-ray diffraction analysis, assuming a biaxial stress state model. Within the experimental accuracy of their studies they found that the internal stresses were small in magnitude ( 1 to $2 \mathrm{GPa}$ ), tensile in nature and independent of growth temperature for thin films of the order of a few microns. In films of $10 \mu \mathrm{m}$ thickness, removing the substrate to produce a membrane structure resulted in considerable stress release. They also demonstrated that in these thicker films a stress gradient existed within the coating.

Berry et al(5) have measured the tensile stress in membranes formed from CVD diamond films deposited onto silicon using a vibrating-membrane method. The resonance of a circular membrane is excited and detected electrostatically and the relationship between stress $\sigma$ and the frequencies, $f_{n}$, is

$$
f_{n}=C_{n} / b(\sigma / \rho)^{1 / 2}
$$

where $\mathrm{C}_{\mathrm{n}}$ is the mode constant, $\mathrm{b}$ the membrane diameter and $\rho$ the density. At room temperature stresses were found to lie in the range $9.4-138 \mathrm{MPa}$ and the projected stresses at the growth temperature $(1123 \mathrm{~K})$ were estimated to be $450-620 \mathrm{MPa}$. The smaller variation in stresses at high temperature lead Berry et al (7) to deduce that the room temperature stress arises from the opposing influence of a tensile growth stress and a thermal stress of comparable and opposite magnitudes (500 MPa). This observation suggests that factors influencing the growth stress should therefore have a marked effect on the magnitude and sign of the room temperature stress.

This paper describes how two techniques Raman spectroscopy (band shifts) and XRD $\left(\sin ^{2} \psi\right.$ method) have been used to measure the residual stresses in diamond films deposited in this Laboratory. The various contributions to growth and thermal stresses within these films is discussed.

\section{Experimental}

Diamond films were deposited on silicon and alumina substrates using thermal filament and microwave assisted CVD processes. To facilitate diamond growth the substrates were polished by mechanically abrading their surfaces with $1 \mu \mathrm{m}$ diamond powder prior to deposition. Methane $(0.5$ $0.8 \%$ ) and hydrogen (diluent) were the main source gases, however oxygen was added to facilitate the MACVD process. The substrates were maintained at temperatures ranging from $500-1000{ }^{\circ} \mathrm{C}$ and the total pressure was kept constant at approximately 50 mbar, during deposition.

$X$ - ray diffraction studies were made to determine the residual stresses within the diamond films using the $\sin ^{2} \psi$ method (11), which in its simplest form assumes that a biaxial stress state exists within the coating. The method uses the interplanar spacing as an internal strain gauge, measuring the strain along a particular direction. A fuller description of the $\sin ^{2} \psi$ method is given by Chollet, Boving and Hintermann (12).

Laser Raman spectroscopy was performed on the diamond films using the Harwell laser Raman microprobe (13), with a spatial resolution of $1-2 \mu \mathrm{m}$. The $514.531 \mathrm{~nm}$, green line of an $\mathrm{Ar}^{+}$laser was used to illuminate a sample held in a microscope hot stage accessory, which was capable of heating a sample to $750^{\circ} \mathrm{C}$ in a controlled atmosphere. The $180^{\circ}$ backscattered light was collected via a high numerical aperture, extra long working distance $40 \mathrm{x}$ objective lens and passed to a Spex Triplemate spectrometer fitted with a 1024 element photodiode array detector. 


\section{Results and Discussion}

Typical first order Raman spectra of diamond films grown at 700,800 and $900^{\circ} \mathrm{C}$ on silicon substrates can be seen in Figure 1. This shows the characteristic sharp band at $1332 \mathrm{~cm}^{-1}$, due to scattering from diamond centres and a broad feature at $1550 \mathrm{~cm}^{-1}$ due to $\mathrm{sp}^{2}$ bonded amorphous or graphitic carbon (14).
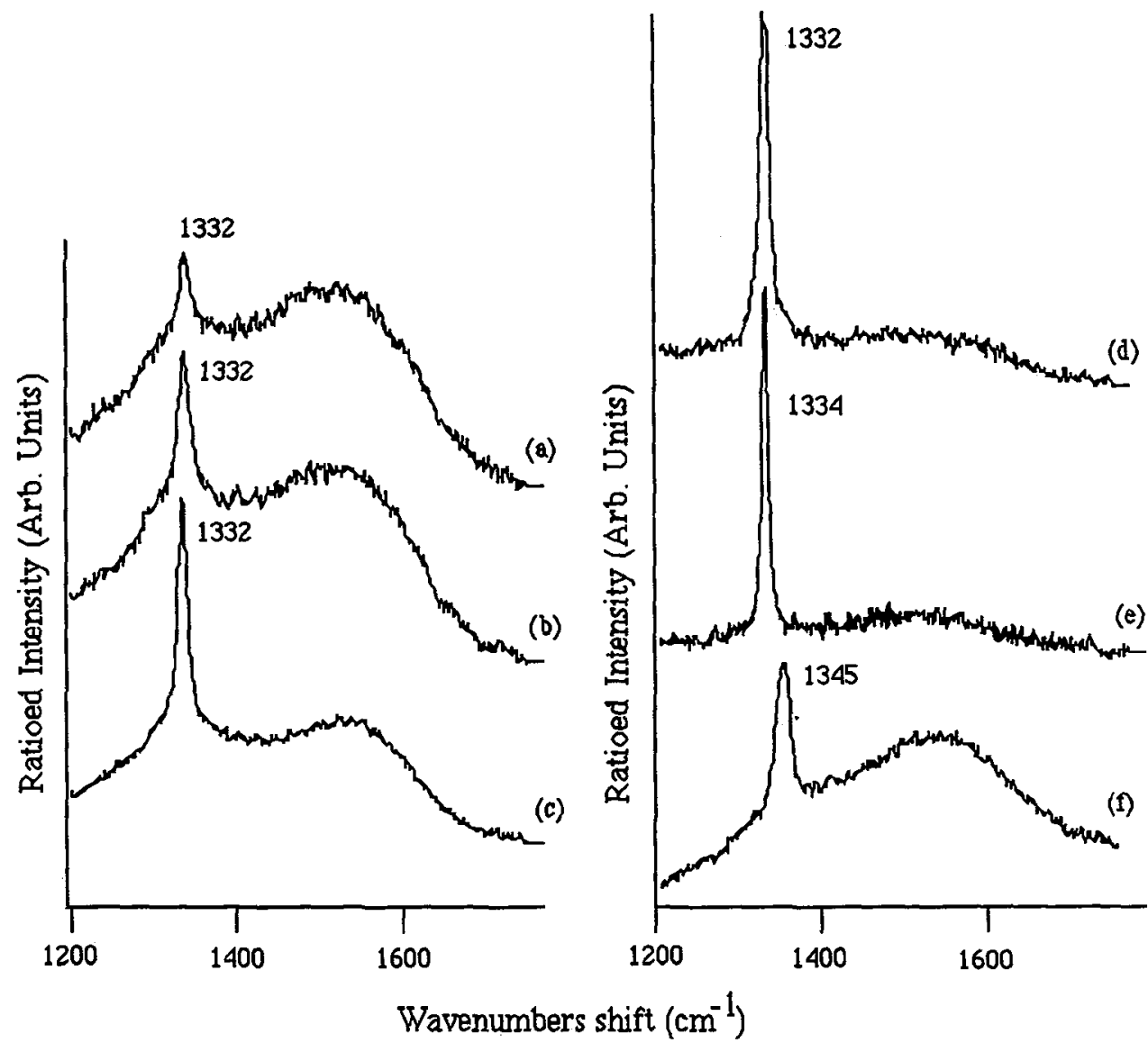

Figure 1. Raman spectra obtained from diamond films deposited at (a) 700, (b) 800 and (c) $900^{\circ} \mathrm{C}$. High resolution Raman spectra were recorded from diamond films deposited on silicon substrates using (d) TFCVD and (e) MACVD and on alumina substrates using MACVD (f).

$\mathrm{X}$-ray stress analysis of these films was performed assuming a biaxial stress state and a residual stress component $\sigma_{\phi}$ was derived (where $\sigma_{\phi}=\sigma_{11} \cos ^{2} \phi+\sigma_{22} \sin ^{2} \phi$ ) which in each case was tensile in nature. Values of $\sigma_{\phi}$ for each substrate temperature are given in Figure 2 . Within the experimental accuracy of this procedure the stress values are comparable indicating that the tensile stress is relatively insensitive to the substrate growth temperature. This result is to be expected when the observed stress, $\sigma_{\mathrm{obs}}$, can be expressed as the sum

$$
\begin{array}{ll}
\sigma_{\mathrm{obs}} & =\sigma_{\text {thermal }}+\sigma_{\text {intrinsic }} \\
\text { or, } \quad \sigma_{\mathrm{obs}}=\Delta \alpha \Delta \mathrm{T}\left(\mathrm{E}_{\mathrm{f}} /\left(1-\mathrm{v}_{\mathrm{f}}\right)\right)+\sigma_{\text {intrinsic }}
\end{array}
$$

where, $E_{f}$ is the Young's modulus and $v_{f}$ is the Poisson's ratio of the material in question. 
where $\Delta \alpha$ is the difference in coating and substrate thermal expansion coefficients and $\Delta \mathrm{T}$ is the difference between deposition temperature and room temperature. It is estimated that at the extremes of growth temperature only a factor of 1.3 difference is expected in the thermal stress components.

The lattice mismatch between the substrate and the diamond film would be expected to introduce a local stress at the coating-substrate interface and the contribution of this parameter to the internal stresses of the diamond film has been investigated previously $(10)$ by the analysis of a diamond membrane and thick diamond films. Form this work a model has been developed to explain the stress distribution found in diamond films. The model is based on the premise that large tensile stresses dominate at the coating-substrate interface due to lattice mismatch between the diamond and the silicon but as the film grows outwards, a balancing compressive stress develops with thickness.

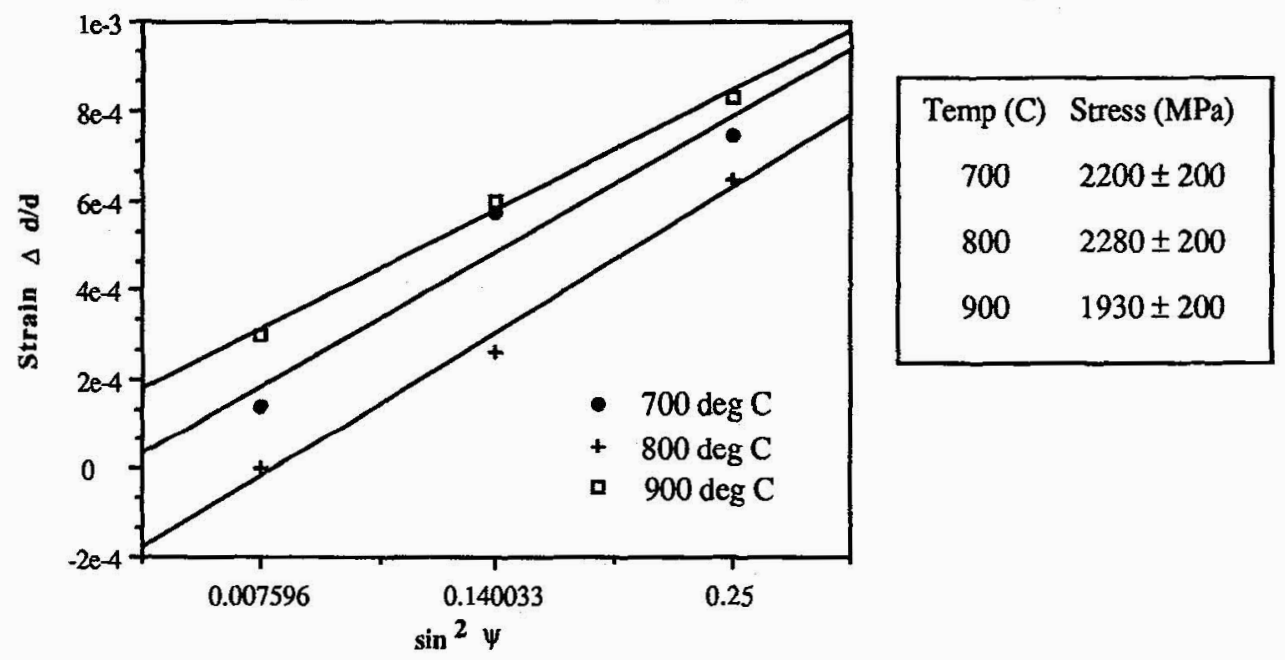

Figure 2. Residual stress components for three substrate temperatures, dervived from XRD measurements.

The cross-sectional SEM pictures in Figure 3 show that the diamond films grow with a columnar microstructure and in the case of these films deposited by unbiased thermal filament CVD considerable porosity exists at the interface. At the growth temperature the columnar structures widen as the film thickens and on cooling to room temperature the difference in thermal expansion coefficients between the silicon and diamond would cause the upper parts of the columns to be put under compressive stress.

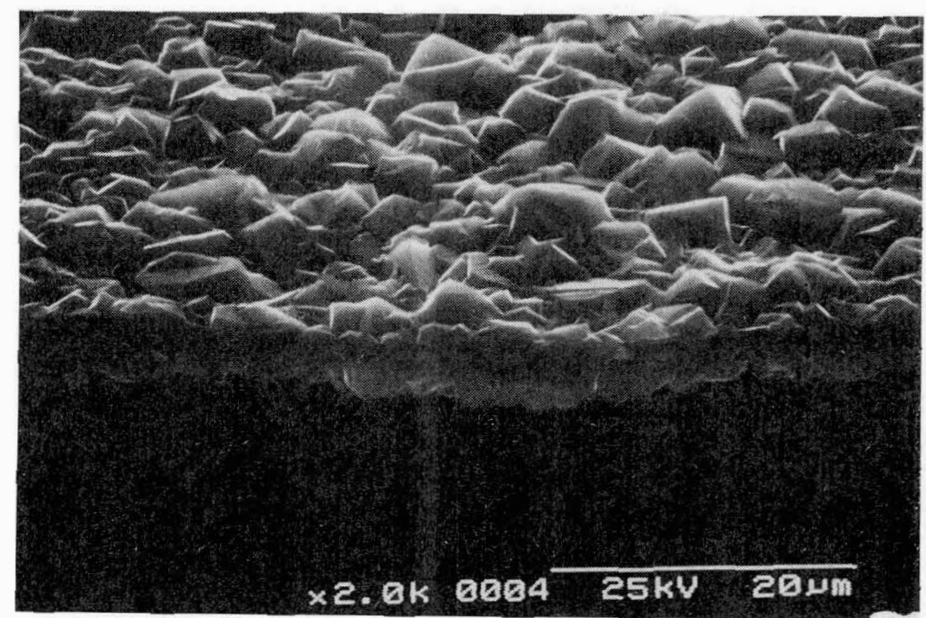

Figure 3. Cross sectional SEM image of a diamond film deposited bv TFCVD 
To extend and verify this model further, diamond films were grown on alumina substrates using a microwave assisted CVD process. This allowed two tests of the model:

(i) alumina has a thermal expansion coefficient 20 times that of silicon, and;

(ii) using an MACVD process a greater nucleation density could be achieved.

Diamond films on alumina deposited by MACVD typically display crystallites of sub-micron sizes, whereas TFCVD films generally have crystallites in the range of 1 to $5 \mu \mathrm{m}$. From this observation we can deduce that the nucleation density is higher in MACVD processes. This may be due to the higher concentration of free hydrogen radicals and extended supersaturation of reactive hydrocarbon precursors encountered in the hotter MACVD plasma system. The high concentration of hydrogen radicals opens up active deposition sites by abstraction of bound hydrogen from the growing surfaces, so promoting a reduced surface energy, which decreases the critical nucleus size necessary for nucleation and growth.

A typical Raman spectrum obtained from a diamond film on an alumina substrate can be seen in Figure 1. Notice that the characteristic Raman band due to diamond has now shifted to higher frequency $\left(1345 \mathrm{~cm}^{-1}\right)$ due to the severe stress that the film has been subjected to. These large shifts in the Raman spectra are easily measured, allowing the stresses to be measured accurately. This is fortunate since the use of polycrystalline alumina substrates presents considerable problems when using XRD methods for determining stresses in diamond films, due to overlapping reflections. Raman spectroscopy also offers the added advantage that spectra can be recorded as a function of temperature and so stress measurements can be performed in the region of the growth temperatures. This, in fact, has been done for diamond films deposited on both alumina and silicon substrates and as a control, $1 \mu \mathrm{m}$ diamond grit.

Any analysis of the stresses in polycrystalline materials by Raman spectroscopy must take into account the effects of finite crystal size. Le Grice et al. (15) have developed a theory of microcrystalline domain size which states simply that the Raman peak from a crystal of finite size will exhibit a smaller Raman shift and have a larger full width half maximum (FWHM) than that from an infinite crystal lattice. This assumes that with the breakdown in selection rules caused by the finite crystal size, the dispersion curves are neither changed nor any new modes created, and that the phonon excited in the lattice by the incident photon is confined to the microcrystalline domain in which it originated.

Using this theory, the effects of frequency shift due to microcrystalline domain size and that due to stress can be deconvoluted, viz:

$$
\Delta \omega_{\text {obs }}=\Delta \omega_{\text {finite }}+\Delta \omega_{\text {stress }}
$$

The contribution arising from a finite crystal size effect is determined from the observed linewidth (FWHM) of the diamond band in the Raman spectrum. The difference between this calculated shift and the observed shift is the shift due to residual stress in the film. The residual stress, $\Delta \omega_{\text {stress, }}$, can be further resolved into two components: one that takes into account the thermal stress, due to differences in coefficients of thermal expansion; and the other which is due to intrinsic stress, arising from the differences in lattice mismatch.

$$
\Delta \omega_{\text {stress }}=\Delta \omega_{\text {thermal }}+\Delta \omega_{\text {lattice }}
$$

Consider the Raman spectra obtained from diamond films deposited on alumina and silicon substrates (Figure 1). The amount of $\mathrm{sp}^{2}$ bonding in the films does not vary significantly (note that the increase in background signal from the diamond on alumina is due to scattering from the alumina substrate itself) but there is a variation in the linewidths (FWHM) of the diamond band. We can use this linewidth $(\Gamma)$ to determine a domain size, which then gives a frequency shift due to a finite crystal size. Corrected frequency shifts for the various diamond films can then be calculated and using the pressure dependence curve of Sharma et al. (16), who plotted the position of the diamond band as a function of applied uniaxial pressure, we can obtain a measure of the internal stress of the diamond films. This is tabulated in Table 1.

It is clear from Table 1 that after having taken into account the microcrystalline domain effect, the diamond film deposited by the TFCVD process is in tension, confirming the XRD measurements. The result from the two techniques does not agree exactly because the accuracy of the line measurements on the Raman microprobe is $0.1 \mathrm{~cm}^{-1}$ on linewidth and some $1.1 \mathrm{~cm}^{-1}$ on line position. Using MACVD, on the other hand, results in compressive stresses being generated in the diamond films deposited on silicon substrates and even larger compressive stresses in those films deposited on alumina substrates. 
These results corroborate the model outlined above. By increasing the nucleation density one reduces the available space each crystallite has to grow in. Thus large compressive stresses are introduced as the crystallites coalesce and grow outwards. These compressive stresses outweigh the tensile stresses which build up at the interface due to lattice mismatch. This is accentuated by utilising alumina substrates, in which case there would be no lessening of interfacial tension between the substrate and the coating due to the formation of a carbide interlayer as could happen with silicon as a substrate, and the large mismatch in thermal expansion coefficients between alumina and diamond would introduce large compressive thermal stresses as the films were cooled.

\section{Table 1}

\section{Stress calculations and related values for diamond films}

$\begin{array}{clccccc}\text { Substrate } & \text { Method } & \Gamma\left(\mathrm{cm}^{-1}\right) & \text { Domain }(\AA) & \Delta \omega_{\text {obs }}\left(\mathrm{cm}^{-1}\right) & \Delta \omega_{\text {stress }}\left(\mathrm{cm}^{-1}\right) & \text { Stress }(\mathrm{GPa}) \\ \mathrm{Si} & \text { TFCVD } & 4.1 & 190 & -0.5 & -1.3 & -0.55 \\ \mathrm{Si} & \text { MACVD } & 5.2 & 118 & 1.1 & 2.6 & 1.1 \\ \mathrm{Al}_{2} \mathrm{O}_{3} & \text { MACVD } & 9.2 & 76 & 12.6 & 9.9 & 4.2\end{array}$

The frequency shifts of the diamond Raman band for a number of films on both alumina and silicon substrates as well as diamond grit have been plotted as a function of temperature and can be seen in Figure 4. The films were heated in both hydrogen and an inert atmosphere of argon. As predicted by theory (17) there is a softening of the TO (X) mode $\left(1332 \mathrm{~cm}^{-1}\right)$ of diamond to lower frequency shifts with increasing temperature. This shift can be seen in Figure 4 by following the plot of the diamond grit, which acts as a standard for the experiment. Some spectral resolution had to be lost in order to gain enough sensitivity to be able to perform the analysis at elevated temperatures, and as a result there is considerable scatter in the data.

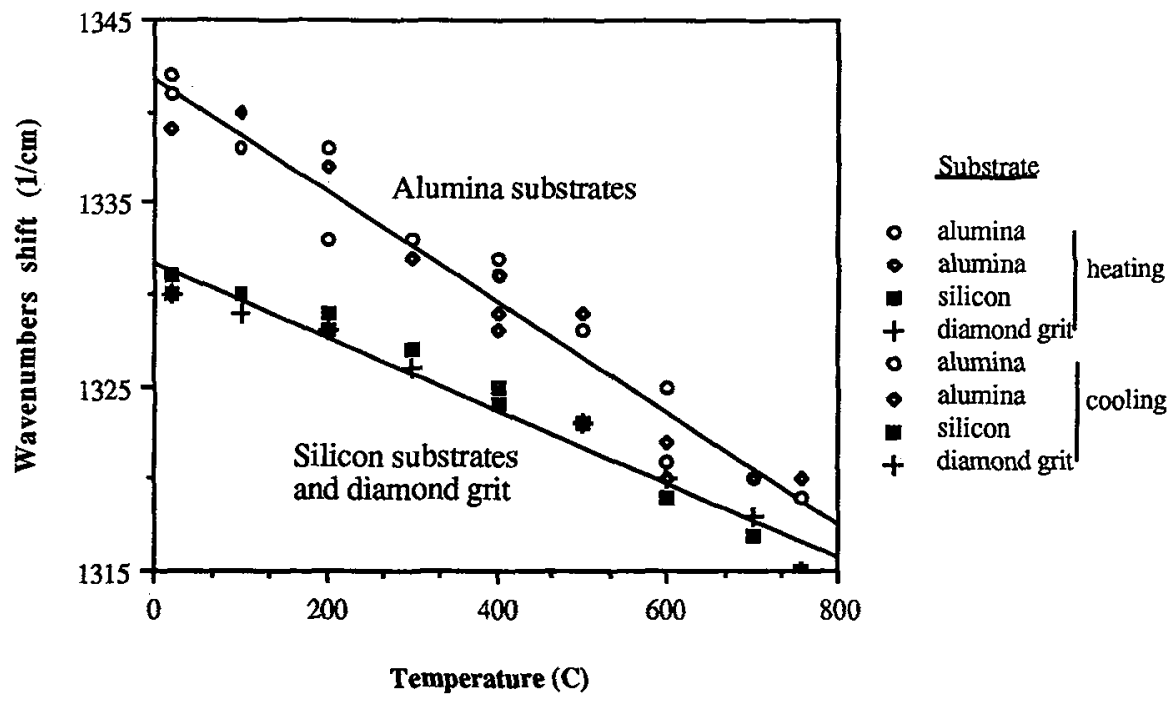

Figure 4. Diamond Raman band position as a function of temperature.

However, it is clear that the diamond films deposited on silicon follow the diamond grit plot closely, while the diamond films deposited on alumina substrates display a totally different behaviour. Within experimental error we can say that the diamond films deposited on silicon are relatively unstressed throughout the temperature range studied. On the other hand, those films deposited on alumina substrates show considerable compressive stress, which relaxes, ie. tends towards the diamond grit plot, as the temperature approaches that of the growth temperature. By projecting the data to the actual growth temperature of $850^{\circ} \mathrm{C}$, we can see that the films deposited on alumina are unstressed at the actual growth temperatures, which is to be expected. 


\section{Conclusions}

The internal stresses in diamond films has been investigated using the XRD $\sin ^{2} \psi$ method and by laser Raman microprobe spectroscopy. They have been measured as a function of substrate material (silicon and alumina), nucleation density (TFCVD and MACVD) and temperature (room temperature to $750^{\circ} \mathrm{C}$ ), and the results used to verify a model proposed by Chalker et al.

By increasing the nucleation density, diamond films can be deposited which display internal stresses which are compressive in nature. These stresses are increased even further by increasing the lattice mismatch between the substrate and the diamond coating. This compressive stress component is attributed to the difference in thermal expansion coefficients between the coating and the substrate and as the films are cooled from their growth temperature the columnar microstructure is compressed.

It may therefore be possible to produce diamond thin film devices, using a high nucleation density growth method such as MACVD at temperatures at which the device is designed to operate, by compensating for residual stresses one promotes adhesion at elevated operating temperatures.

This work is continuing with the application of the XRD $\sin ^{2} \psi$ method to analyse stresses in diamond films on silicon at high temperatures.

\section{Acknowledgements}

The authors would like to acknowledge the provision of funding by the Corporate Research Programme of AEA Technology and Dr. K. Kobashi of Kobe Steel Ltd., Japan for supplying some of the diamond films on alumina.

\section{References}

(1) J.E. Field, The Properties of Diamond, Acadamic Press, 1979.

(2) P. K. Bachmann, W. Drawl, D. Knight, R. Weimer and R. F. Messier, Diamond and Diamond - Like Materials Synthesis, eds., G. H. Johnson, A. R. Badzian and M. Geis, Mater. Res, Soc., 1988.

(3) M.Yoshikawa, H.Ishida, A.Ishitani, T.Murakami,, S. Koizumi and T.Inuzuka Appl. Phys.Letts 57 (1990) 428.

(4) G. Jansen, W. Volenberg, L. J. Giling, W. J. P. van Enckevort, J. J. D. Schaminee and M. Seal, Porc De Beers Diamond Conf., Reading, 1990.

(5) B.E. Williams, H.S.Hong and J.T. Glass J. Mater. Res.5 (1990) 801.

(6) R,W.Bower Sensors and Actuators A21-A23 (1990) 993.

(7) B.S.Berry, W.C. Pritchet, J.J. Cuomo, C.R. Guarnieri and D.J.Whitehair Appl. Phys Lett 57 (1990) 302.

(8) H.Boppart, J. van Straaten and I.F. Silvera, Phys. Rev. B, 32 (1985) 1423.

(9) M.Yoshikawa, G.Katagiri, H.Ishida, A Ishitani, M.Ono and K.Matsumura Appl. Phys. Lett., 55 (1989) 2608.

(10) P. R. Chalker, A. M. Jones, C. Johnston and I. M. Buckley-Golder, presented Diamond Films '90, Crans - Montana, Switzerland, September, 1990. To be published Thin Films Technol., 1991.

(11) B.D. Cullity, Elements of X-ray Diffraction, Addison-Wesley, Reading, MA, 1978, 2nd Edn., pp. 447-479.

(12) L. Chollet, H. Boving and H. E. Hintermann, J. Mater. Energy Syst., 6 (1985) 293.

(13) P. R. Graves, UKAEA Report, AERE R 11698 (1985).

(14) C. Johnston, UKAEA Report, AEA - InTec - 0198 (1990).

(15) Y.M LeGrice, R.J.Nemanich, J.T.Glass, Y.H.Lee, R.A.Rudder and R.J. Markunas appeared in Mat. Res. Symp., Vol. 162.

(16) S. K. Sharma, H. K. Mao, P. M. Bell and J. Z. Xu, J. Raman Spectros., 16 (1985) 350.

(17) C. Z. Wang, C. T. Chan and K. M. Ho, Phys. Rev. B, 42 (1990) 11276. 